ORIGINAL PAPER

\title{
ULTRASTRUCTURAL ALTERATIONS OF THE TYMPANIC MEMBRANE IN RETRACTION POCKETS FROM PATIENTS WITH CHRONIC OTITIS MEDIA
}

\author{
Dmitry Tretiakow ${ }^{1}$, Jerzy Kuczkowski ${ }^{1}$, Cecylia Tukaj ${ }^{2}$
}

${ }^{1}$ Department of Otolaryngology, Medical University of Gdansk, Poland

${ }^{2}$ Department of Electron Microscopy, Medical University of Gdansk, Poland

\begin{abstract}
Chronic otitis media with a retraction pocket is one of the forms of chronic otitis media that predisposes to cholesteatoma. The aim of our study was to determine the extent and type of changes in the tympanic membrane fragments which were surgically excised from the retraction pockets of 20 patients treated for cholesteato$\mathrm{ma}$ and/or hearing loss. Material evaluation and photographic documentation were performed using a transmission electron microscope (JEM 1200EX II, Jeol, Japan). We found that the morphological changes of the tympanic membrane are particularly related to the structure of the external epithelium, formation of epithelial invaginations, atrophy of the basement membrane of the eardrum and loss of circular and longitudinal collagen fibers. Ultrastructural changes in the tympanic membrane suggest inflammatory processes, epithelial migration, destruction of collagen fibers and autophagy, which indicates that the process of retraction pocket development is active. Tympanic membrane retraction pocket development is an active process, not the sequela of prior illness. The intensification of the keratinization and migration process of the epithelium layer confirms one of the theories of the creation of cholesteatoma and shows that the retention pocket is a pre-cholesteatoma condition.
\end{abstract}

Key words: collagen fibers, transmission electron microscopy, retraction pocket, otitis, cholesteatoma.

\section{Introduction}

Chronic otitis media with a retraction pocket is a form of chronic otitis media and can lead to hearing loss and cholesteatoma $[1,2,3,4]$. The causes of tympanic membrane retraction (alternatively described as eardrum atelectasis) and the mechanism of bone destruction are still controversial and not entirely known. Some authors suggest that the development of the tympanic membrane retraction pocket is a passive process that is sometimes activated by several different factors (e.g. dysfunction of the Eustachian tube) $[2,5,6,7,8]$. The ambiguity of the etiopathogenesis of this disease often hinders its effective treatment.

According to the literature, an inflammatory process of the upper respiratory tract leading to disturbed ventilation of the middle ear cavity plays a key role in the development of chronic otitis media with a retraction pocket $[9,10,11,12]$. Local inflammatory reaction determines the onset of the disease and its further course. Extra- and intra-tympanic mechanisms can be distinguished in the literature on the etiopathogenesis of the tympanic membrane retraction pocket. The intra-tympanic mechanism is conditioned by the activity of proteolytic enzymes and cytokines 
found in the inflammatory exudate, whereas proteolytic enzymes found in the inflamed epithelium are responsible for the extra-tympanic mechanism. Lysozyme, hydrolases and pro-inflammatory cytokines located in the outer layer of the tympanic membrane can destroy not only the collagen fibers of its middle layer but also the adjacent bone tissue (e.g. lateral epidermal wall, ossicles). Fortunately, there is a system of antagonistic enzymes that ensure balance. An imbalance in the activity of these enzymes leads to the destruction of all layers of the tympanic membrane and bone tissue, as well as the further development of the retraction pocket and cholesteatoma [9, $10,11,12,13]$.

Our study aimed to determine the extent and type of changes in the tympanic membrane fragment excised from the retraction pocket and to examine the eardrum fibrous elements in patients with a retraction pocket of the tympanic membrane.

\section{Material and methods}

The specimens for ultrastructural studies were dissected intraoperatively from 20 patients (18-45 years old) who were admitted to the department with the pars tensa tympanic membrane retraction pocket (stage II-IV graded according to Sade's classification [9]) in the course of chronic otitis media. We did not have patients with stage I of the retraction pocket, because such patients have no indications for surgical treatment.

After immediate flushing in phosphate-buffered saline (PBS) and cutting into $1-\mathrm{mm}^{3}$ pieces, the obtained samples were placed in a fixing solution $(2.5 \%$ glutaraldehyde in $0.1 \%$ cacodylate buffer at $\mathrm{pH} 7.4$ with the addition of sucrose). Sample fixation was carried out at $4^{\circ} \mathrm{C}$ for $24 \mathrm{~h}$ and afterward, the material was rinsed 3 times in the same buffer. Tissue fragments were then stained for 2 hours in a $2 \%$ osmium tetroxide solution $\left(\mathrm{OsO}_{4}\right.$ in the same buffer) and then washed 3 times in cacodylate buffer. Dehydration of such fixed material takes place in a series of ethanol solutions with increasing concentration (30\%, 50\%, $75 \%, 90 \% 2 \times$ and absolute ethanol). The material was additionally immersed in propylene oxide. The samples were embedded in a several-stage epoxy resin (Epon 812, Merck) and then polymerized at $37^{\circ} \mathrm{C}$.

Semi-thin sections $(1.5 \mu \mathrm{m})$ were obtained with a glass knife on an Om U2 ultramicrotome (Richert, Austria) and then stained with toluidine blue and evaluated by light microscopy. Ultrathin sections (60-80 nm) were obtained using a diamond knife and were collected into Formvar-covered copper grids. Sections then were contrasted with uranyl acetate and lead citrate.
Material evaluation and photographic documentation were performed using a transmission electron microscope (JEM 1200EX II, Jeol, Japan) at $80 \mathrm{kV}$. The collection of over 600 images allowed for repeated, consistent assessment of the material, comparison of obtained results and formulation of conclusions.

The biological material was collected from adults because the development of retraction pockets in children is often more dynamic and other factors affect their development. Also, this age range (18-45 years) was chosen because these patients are often treated conservatively in the first stage of the tympanic retraction pocket.

\section{Results}

Due to the sparse literature data on tympanic membrane ultrastructure, we undertook this analysis on our own material (Table I).

Ultrastructural analysis of the obtained material revealed morphological changes of the external epithelium structure, formation of epithelial invaginations, atrophy of the basement membrane of the eardrum and loss of circular and longitudinal collagen fibers. The obtained data also indicate intensified inflammatory processes, epithelial migration, destruction of collagen fibers and autophagy. In the more advanced retraction pockets (stage III-IV), the morphological changes were slightly more pronounced compared to the stage II retraction pockets.

The intense epithelial keratinization attracts attention. We observed elongated bodies of keratinocytes with an unusual arrangement (parallel to the basement membrane) which may indicate cell migration (Fig. 1A, B). Of note in this image is the evident invagination of the epithelium in loose tissue of the lamina propria (Fig. 1A).

Extravasated erythrocytes present in the field of view. Capillary endothelial cells have evident degenerative features (Fig. 2B). Infiltration with leukocytes indicates the presence of an inflammatory process (Fig. 2A). Similar changes were observed in the majority of samples tested.

The chaotic arrangement of collagen fibers and the features of various degrees of their degradation indicate an advanced degenerative process in the circular membrane layer (lamina propria) in the area of the retraction pocket (Fig. 3A, B).

Ultrastructural features of necrotic connective tissue cell changes such as cytoplasmic vacuolization and membrane degradation have been observed. Clusters of collagen fibers with varying degrees of degradation were found (Fig. 3B; 4A,B).

Ultrastructural features of degradation of cellular elements in the area of connective tissue indicate an advanced process of autophagy (Fig. 5). 
Table I. Patient clinical data and descriptive data of changes observed in the retraction pocket in TEM

\begin{tabular}{|c|c|c|c|c|}
\hline $\mathbf{N}$ & SEX & AGE & $\begin{array}{l}\text { RETRACTION } \\
\text { POCKET STAGE }\end{array}$ & DESCRIPTIVE DATA \\
\hline 1 & $\mathrm{~m}$ & 43 & II & $\begin{array}{c}\text { Formation of epithelial invaginations, destruction of collagen fibers, inflammatory } \\
\text { process signs }\end{array}$ \\
\hline 2 & $\mathrm{~m}$ & 18 & III & $\begin{array}{l}\text { Formation of epithelial invaginations, atrophy of the basement membrane, loss } \\
\text { of circular and longitudinal collagen fibers, epithelial migration, inflammatory } \\
\text { process signs }\end{array}$ \\
\hline 3 & $\mathrm{~m}$ & 32 & II & $\begin{array}{c}\text { Formation of epithelial invaginations, atrophy of the basement membrane, } \\
\text { autophagy, inflammatory process signs }\end{array}$ \\
\hline 4 & $\mathrm{w}$ & 31 & II & $\begin{array}{l}\text { Formation of epithelial invaginations, epithelial migration, destruction of collagen } \\
\text { fibers, inflammatory process signs }\end{array}$ \\
\hline 5 & $\mathrm{~m}$ & 27 & II & Formation of epithelial invaginations, inflammatory process signs \\
\hline 6 & $\mathrm{w}$ & 24 & II & Formation of epithelial invaginations, inflammatory process signs \\
\hline 7 & $\mathrm{~m}$ & 34 & III & $\begin{array}{c}\text { Formation of epithelial invaginations, atrophy of the basement membrane, loss } \\
\text { of circular and longitudinal collagen fibers, autophagy, inflammatory process } \\
\text { signs }\end{array}$ \\
\hline 8 & $\mathrm{w}$ & 28 & III & Formation of epithelial invaginations, atrophy of the basement membrane \\
\hline 9 & $\mathrm{~m}$ & 42 & IV & $\begin{array}{l}\text { Formation of epithelial invaginations, atrophy of the basement membrane, } \\
\text { destruction of collagen fibers, inflammatory process signs }\end{array}$ \\
\hline 10 & $\mathrm{w}$ & 24 & III & Formation of epithelial invaginations, autophagy \\
\hline 11 & $\mathrm{w}$ & 37 & II & Formation of epithelial invaginations, atrophy of the basement membrane \\
\hline 12 & $\mathrm{w}$ & 25 & III & $\begin{array}{l}\text { Formation of epithelial invaginations, epithelial migration, autophagy, } \\
\text { destruction of collagen fibers, inflammatory process signs }\end{array}$ \\
\hline 13 & $\mathrm{~m}$ & 37 & II & Formation of epithelial invaginations \\
\hline 14 & $\mathrm{~m}$ & 41 & III & Formation of epithelial invaginations, epithelial migration \\
\hline 15 & $\mathrm{w}$ & 33 & II & $\begin{array}{l}\text { Formation of epithelial invaginations, atrophy of the basement membrane, } \\
\text { destruction of collagen fibers, inflammatory process signs }\end{array}$ \\
\hline 16 & $\mathrm{~m}$ & 23 & III & $\begin{array}{l}\text { Formation of epithelial invaginations, atrophy of the basement membrane, loss } \\
\text { of circular and longitudinal collagen fibers, epithelial migration, autophagy, } \\
\text { inflammatory process signs }\end{array}$ \\
\hline 17 & $\mathrm{w}$ & 44 & II & $\begin{array}{l}\text { Formation of epithelial invaginations, atrophy of the basement membrane, } \\
\text { inflammatory process signs }\end{array}$ \\
\hline 18 & $\mathrm{~m}$ & 43 & II & Formation of epithelial invaginations \\
\hline 19 & $\mathrm{w}$ & 39 & IV & $\begin{array}{l}\text { Formation of epithelial invaginations, atrophy of the basement membrane, loss } \\
\text { of circular and longitudinal collagen fibers, epithelial migration, autophagy }\end{array}$ \\
\hline 20 & $\mathrm{~m}$ & 45 & II & Formation of epithelial invaginations, autophagy, inflammatory process signs \\
\hline
\end{tabular}

\section{Discussion}

Our observations have shown that the formation of the retraction pocket is accompanied by deformation of the tympanic membrane. We observed significant changes in epidermal and fibrous layers in the retraction pocket of their histological structure and at the subcellular level of organization. These changes particularly relate to the outer epithelium (epidermal layer) and the lamina propria.

The epidermal layer, which is the lining of the retraction pocket, lost its characteristic layering. Furthermore, its cells changed morphological features; thus the epithelial layers (basal, spinous and granu- lar) are practically indistinguishable. In consequence, the basal layer, circular and longitudinal fiber layer and epithelial layer are practically indistinguishable.

Epithelial keratinocytes most often had an elongated shape regardless of their location in the assessed material. Their arrangement was often chaotic and the number of characteristic connections between the desmosomes was reduced. Relatively few intermediate filaments (tonofilaments) were present in the cytoplasm. Electron microscopy images revealed disruption of the basement membrane of epithelial cells, its atrophy or disturbed structure, a decrease of the number of hemidesmosomes, and numerous pseudopodia enabling movement of the cell in 

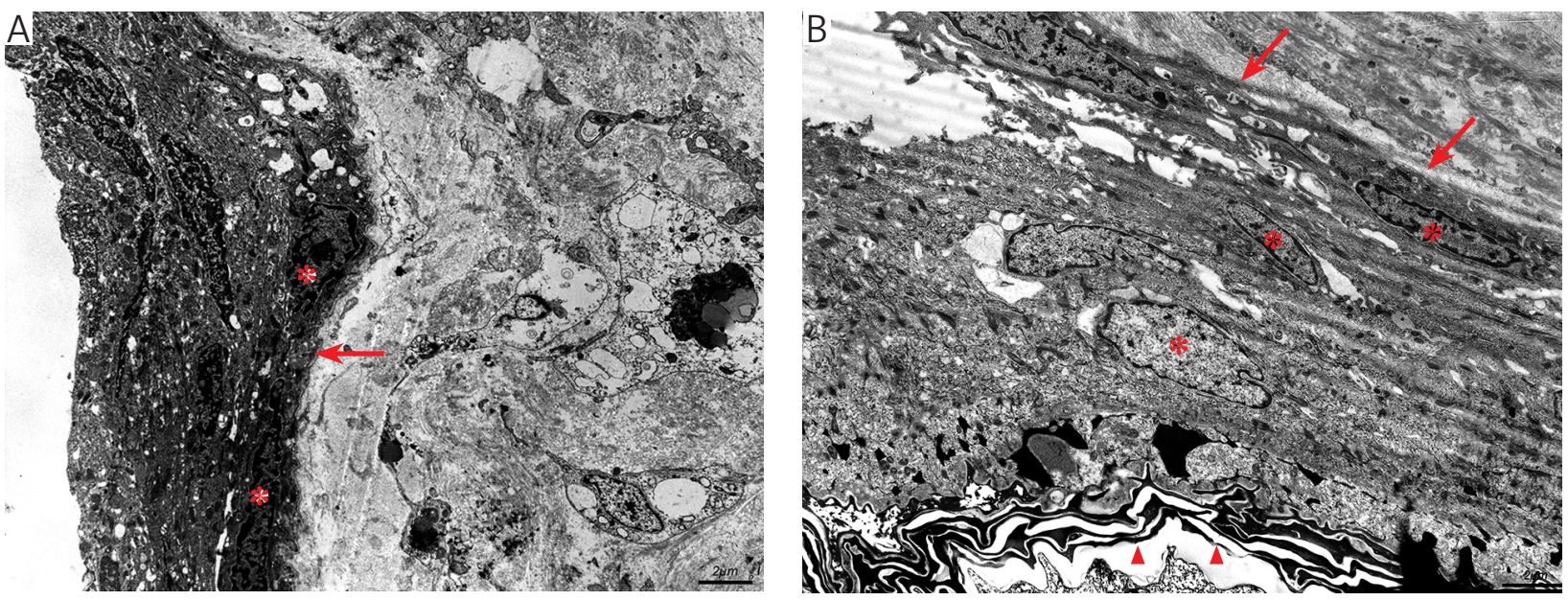

Fig. 1. Ultrastructure of the retraction pockets in electron microscopy. A) The stratified squamous epithelium and the connective tissue of lamina propria (underneath the epithelium). The nucleus $(*)$, basement membrane ( $\uparrow$ ). B) Epidermal layer of the retraction pockets in the electron microscopy. The basement membrane $(\uparrow)$, the nucleus $(*)$, the cornified layer of the epithelium $(\Delta)$
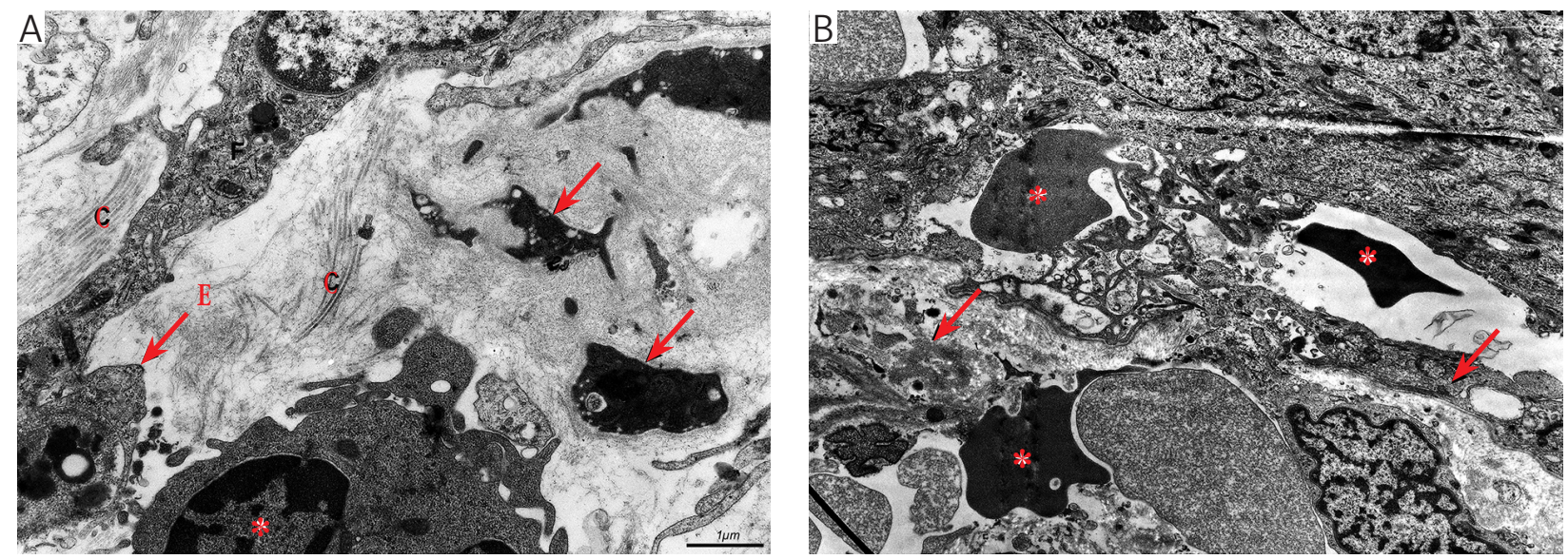

Fig. 2. The ultrastructure of the connective tissue under the outer layer of epithelium. A) The fragment of a fibroblast (F) with well-preserved cellular organelles and cross-sections of cells $(\uparrow)$ in different stages of degradation. Infiltration of blood elements (leukocyte nucleus $(*)$ marks the presence of inflammation. Collagen fibers (C). B) Extravasated erythrocytes (*), collagen fibers $(\uparrow)$ and endothelial cells (E)
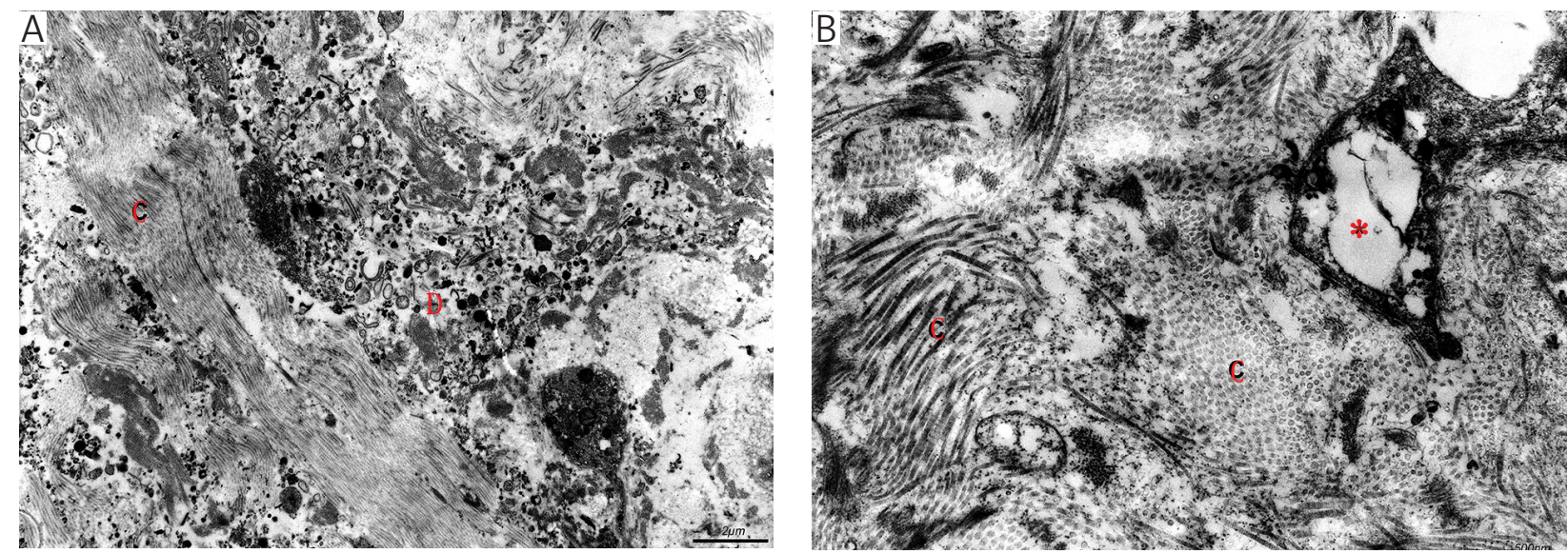

Fig. 3. A) The formation and arrangement of collagen fibers (C). Cellular debris (D) are in the middle part of the field of view. B) Chaotic arrangement of collagen fibers (C) is visible in the longitudinal and oblique sections. Vacuolisation of the cytoplasm (*) and degradation of membranous structures of a cell in the right part of the field 

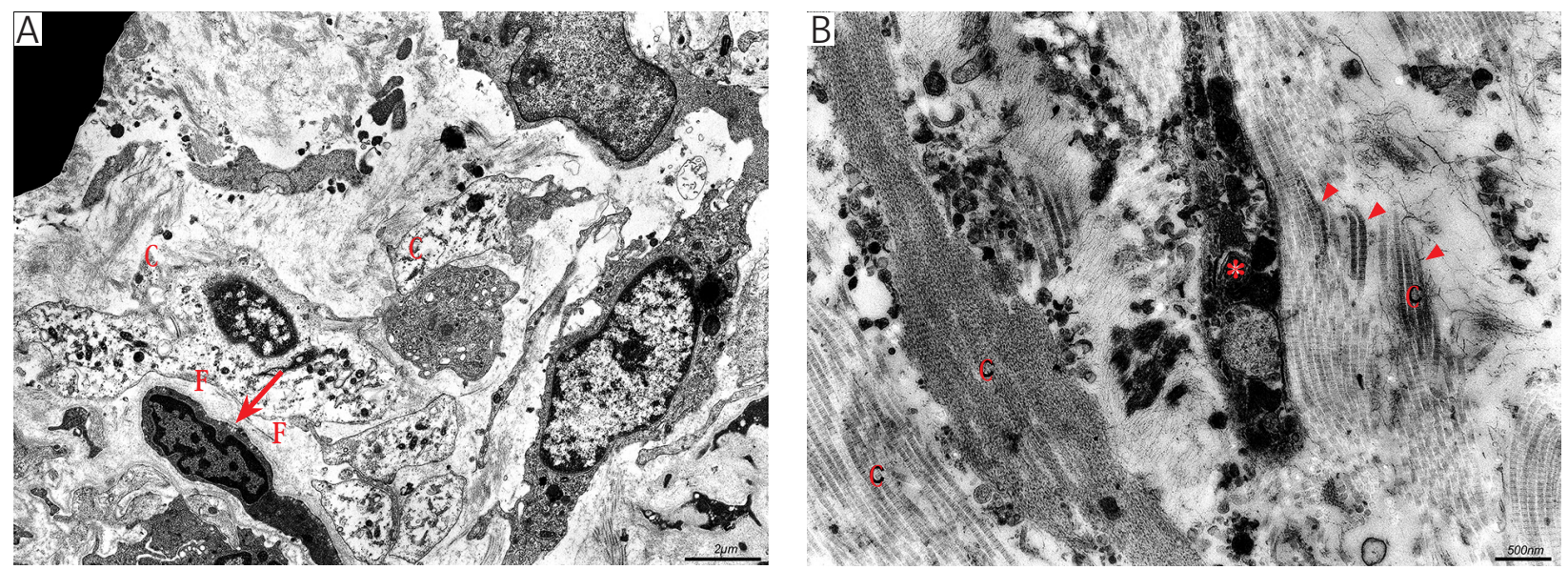

Fig. 4. A) The ultrastructural features of necrotic changes of the connective tissue in retraction pockets. Fibroblastic cell nucleus $(*)$, numerous pinocytic vesicles of fibroblast $(\uparrow)$, newly created collagen fibers $(\mathrm{F})$, the collagen fibers of different degradation level (C). B) The collagen fibers (C) in retraction pocket. Magnification of the characteristic striations of fibers $(\Delta)$. Fragments of the cells with necrosis features $(*)$ are visible in the middle of the image

different directions. This indicates that the epithelial keratinocytes of the retraction pockets disturbed the process of cell differentiation and development of characteristics in the particular layer. Lack of cell differentiation features and the parallel orientation to the basement membrane may be associated with the cells' enhanced migration. However, it is a natural phenomenon for the epithelial cells, which cover the tympanic membrane from the external auditory canal side, to migrate $[14,15]$. In the case of retraction pockets, we observed an intensification of the epithelial cell migration process. Another important and interesting feature that was observed in the examined material was a clear tendency to form epithelial invaginations in the direction of the adjacent mucosa. Several authors have emphasized the important role of these structures in the pathogenesis of the retraction pocket and then the formation of cholesteatoma $[16,17,18,19,20]$. An intensive keratinization process was also observed in the assessed material.

In the connective tissue under the epidermis, many morphological changes attesting to ongoing degenerative processes could be observed. There were signs of increased autophagy in the fibroblast cytoplasm. Numerous forms of autophagosomes and autophagolysosomes have been observed, as well as excessive vacuolization and loss of cell organelles. In the tissue of the retraction pocket, there were focal features of the ongoing inflammatory process, as evidenced by the local infiltration by immune system cells.

Areas with damaged vascular endothelial cells, resulting in not only exudate but also the extravasation of blood cells, were visible in electron microscopy.

The weakening of the lamina propria collagen fibers is considered to be the direct cause of retraction pocket formation $[9,21,22,23]$. The obtained transmission electron microscope images clearly document

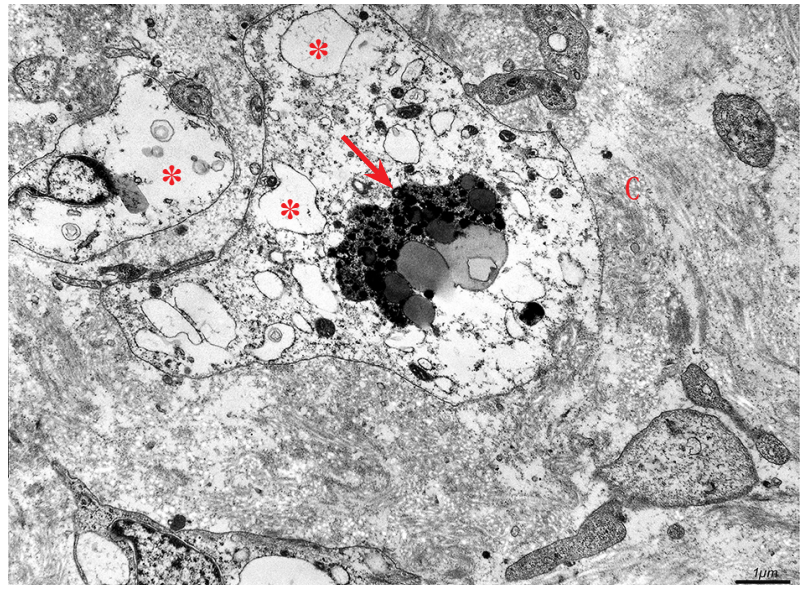

Fig. 5. Ultrastructural features of cellular element degradation in the connective tissue indicate an advanced process of autophagy. Numerous vacuoles contain partially digested material of different electron translucency. Elements of the fibrous intercellular substance are visible in the extracellular space: vacuole $(*)$, autophagosome $(\uparrow)$, collagen fibrils (C)

the disturbing collagen fibers. We observed several features of the collagen fiber degeneration process in varying degrees of intensity caused by their lytic degradation. The characteristic clusters of collagen microfibrils were observed in some areas, thus suggesting an ongoing repair process. Due to the lack of classic clinical features of inflammation (redness, swelling, pain) of the tympanic membrane, it can be assumed that descriptions of random (disorganized) collagen distribution (presented in the figure) are related to the disease itself.

The severity of morphological changes concerning the stage of the retraction pocket may be explained by a disorder of homeostasis mechanisms as a result of worsening inflammation of the middle ear. 
In conclusion, structural changes of the tympanic membrane in the retraction pocket involve the external epithelium and include: formation of epithelial invaginations, atrophy of the basal membrane of the tympanic membrane and the absence of separate circular and longitudinal layers of collagen fibers. The demonstrated ultrastructural changes suggest an intensification of inflammatory processes, epithelial migration, destruction of collagen fibers and autophagy. All of the above indicate the process of retraction pocket development and negate the concept of the retraction pocket as a sequel of a previous disease.

Inflammation activates the extra- and intra-tympanic mechanisms of retraction pocket development. The process of degradation and disorganization of collagen fibers along with the formation of necrotic spaces in the tympanic membrane layers weakens its scaffolding. This in turn predisposes to the invagination of the tympanic membrane towards the tympanic cavity as a result of disturbed tympanic cavity ventilation.

The intensification of the migration process of the epithelium layer confirms one of the theories of the creation of a cholesteatoma and shows that the retention pocket is an active pre-cholesteatoma condition.

We hope that the results of our observations will allow a better understanding of retraction pocket etiopathogenesis and will contribute to the improvement of preventive and therapeutic methods.

The authors declare no conflicts of interest.

This work was supported by departmental funding (ST 02-011/07). The study protocol was approved by the Regional Bioethics Committee of Gdansk Medical University, Poland (approval no. NKBBN/87/2013).

\section{References}

1. Knutsson J, Bagger-Sjöbäck D, von Unge M. Structural tympanic membrane changes in secretory otitis media and cholesteatoma. Otol Neurotol 2011; 32: 596-601.

2. Özkiriş M, Kapusuz Z, Saydam L. The prevalence of middle ear diseases among 7- to 13-year-old primary school students in Yozgat province. Turk J Pediatr 2012; 54: 493-496.

3. Jesic SD, Jotic AD, Babic BB. Predictors for Sensorineural Hearing Loss in Patients With Tubotympanic Otitis, Cholesteatoma, and Tympanic Membrane Retractions. Otol Neurotol 2012; 33: 934-940.

4. Kuczkowski J, Sakowicz-Burkiewicz M, Iżycka-Świeszewska E, et al. Expression of tumor necrosis factor- $\alpha$, interleukin- $1 \alpha$, interleukin-6 and interleukin-10 in chronic otitis media with bone osteolysis. ORL J Otorhinolaryngol Relat Spec 2011; 73: 93-99.

5. Niemczyk K. Kieszonki retrakcyjne - patogeneza i klasyfikacje. 1996.

6. Olszewska E, Wagner M, Bernal-Sprekelsen M, et al. Etiopathogenesis of cholesteatoma. Eur Arch Otorhinolaryngol 2004; 261: 6-24.
7. Grewal DS, Hathiram BT, Moliorikar AV, et al. Retraction pockets in chronic suppurative otitis media - our experience. Indian J Otolaryngol Head Neck Surg 2003; 55: 107-112.

8. Sudhoff H, Tos M. Pathogenesis of sinus cholesteatoma. Eur Arch Otorhinolaryngol 2007; 264: 1137-1143.

9. Sadé J. The buffering effect of middle ear negative pressure by retraction of the pars tensa. Am J Otol 2000; 21: 20-23.

10. D' Eredità R, Marsh RR. Tympanic membrane healing process and biocompatibility of an innovative absorbable ventilation tube. Otol Neurotol 2006; 27: 65-70.

11. Pau HW, Punke C, Just T. Tympanometric experiments on retracted ear drums - does tympanometry reflect the true middle ear pressure? Acta Otolaryngol 2009; 129: 1080-1087.

12. Alzahrani M, Saliba I. Tympanic membrane retraction pocket staging: Is it worthwhile? Eur Arch Otorhinolaryngol 2014; 271: 1361-1368

13. Kobayashi T, Toshima M, Yaginuma Y, et al. Pathogenesis of attic retraction pocket and cholesteatoma as studied by computed tomography. Am J Otol 1994; 15: 658-662.

14. Ala Eddine C, Williams M, Ayache D. Useful imaging of the ear. J Radiol 2006; 87: 1728-1742.

15. Barbara M. Lateral attic reconstruction technique: preventive surgery for epitympanic retraction pockets. Otol Neurotol 2008; 29: 522-525.

16. Boedts D, Decock M, Andries L, Marquet J. A Scanning Electron-Microscopic Study of Different Tympanic Grafts. Am J Otol 1990; 11: 274-277.

17. Boxall JD, Proops DW, Michaels L. Scanning electron microscopy of tympanic membrane epithelium during in vitro migration. J Otolaryngol 1990; 19: 57-61.

18. Kakoi H, Anniko M, Kinnefors A, Rask-Andersen H. Auditory Epidermal Cell Migration. VII. Antigen Expression of Proliferating Cell Nuclear Antigens, PCNA and Ki-67 in Human Tympanic Membrane and External Auditory Canal. Acta Otolaryngol 1997; 117: 100-108.

19. Kakoi H, Anniko M. Auditory epidermal cell migration. V. Transmission electron-microscopic study of tympanic membrane and external auditory canal in the mouse. ORL J Otorhinolaryngol Relat Spec 1996; 58: 280-287.

20. Tang IP, Prepageran N, Raman R, Sharizhal T. Epithelial migration in the atelectatic tympanic membrane. J Laryngol Otol 2009; 123: 1321-1324.

21. Sadé J. On the function of the pars flaccida: Retraction of the pars flaccida and buffering of negative middle ear pressure. Acta Otolaryngol 1997; 117: 289-292.

22. Kutz JW Jr, Fayad JN. Tympanic membrane atelectasis. Ear Nose Throat J 2006; 85: 298.

23. Shu MT, Wu KC, Chen YC. Middle ear atelectasis. Ear, Nose Throat J 2012; 91: 466.

\section{Address for correspondence}

\section{Dmitry Tretiakow}

Department of Otolaryngology

Medical University of Gdansk

Smoluchowskiego 17

80-214 Gdansk, Poland

tel. +48583493110

e-mail: d.tret@gumed.edu.pl 